\title{
The haloarchaeal MCM proteins: bioinformatic analysis and targeted mutagenesis of the $\beta 7-\beta 8$ and $\beta 9-\beta 10$ hairpin loops and conserved zinc binding domain cysteines
}

\section{Tatjana P. Kristensen ${ }^{1+\neq}$, Reeja Maria Cherian ${ }^{1+\neq}$, Fiona C. Gray ${ }^{1}$ and Stuart A. MacNeill ${ }^{1,2 *}$}

1 Department of Biology, University of Copenhagen, Københavns Biocenter, Copenhagen N, Denmark

${ }^{2}$ School of Biology, University of St. Andrews, North Haugh, St. Andrews, Fife, UK

Edited by:

R. Thane Papke, University of Connecticut, USA

Reviewed by:

Nils-Kåre Birkeland, University of

Bergen, Norway

Antonio Ventosa, University of

Sevilla, Spain

${ }^{*}$ Correspondence:

Stuart A. MacNeill, School of

Biology, University of St. Andrews,

North Haugh, St. Andrews, Fife,

KY16 9ST, UK

e-mail: stuart.macneill@

st-andrews.ac.uk

${ }^{\dagger}$ Present address:

Tatjana P. Kristensen, Department of Veterinary Disease Biology,

University of Copenhagen,

Frederiksberg C, Denmark;

Reeja Maria Cherian, Institute of

Biomedicine, Sahlgrenska Academy,

University of Gothenburg, Göteborg,

Sweden

FThese authors have contributed

equally to this work.

The hexameric MCM complex is the catalytic core of the replicative helicase in eukaryotic and archaeal cells. Here we describe the first in vivo analysis of archaeal MCM protein structure and function relationships using the genetically tractable haloarchaeon Haloferax volcanii as a model system. Hfx. volcanii encodes a single MCM protein that is part of the previously identified core group of haloarchaeal MCM proteins. Three structural features of the N-terminal domain of the Hfx. volcanii MCM protein were targeted for mutagenesis: the $\beta 7-\beta 8$ and $\beta 9-\beta 10 \beta$-hairpin loops and putative zinc binding domain. Five strains carrying single point mutations in the $\beta 7-\beta 8 \beta$-hairpin loop were constructed, none of which displayed impaired cell growth under normal conditions or when treated with the DNA damaging agent mitomycin C. However, short sequence deletions within the $\beta 7-\beta 8 \beta$-hairpin were not tolerated and neither was replacement of the highly conserved residue glutamate 187 with alanine. Six strains carrying paired alanine substitutions within the $\beta 9-\beta 10 \beta$-hairpin loop were constructed, leading to the conclusion that no individual amino acid within that hairpin loop is absolutely required for MCM function, although one of the mutant strains displays greatly enhanced sensitivity to mitomycin $\mathrm{C}$. Deletions of two or four amino acids from the $\beta 9-\beta 10 \beta$-hairpin were tolerated but mutants carrying larger deletions were inviable. Similarly, it was not possible to construct mutants in which any of the conserved zinc binding cysteines was replaced with alanine, underlining the likely importance of zinc binding for MCM function. The results of these studies demonstrate the feasibility of using $H f x$. volcanii as a model system for reverse genetic analysis of archaeal MCM protein function and provide important confirmation of the in vivo importance of conserved structural features identified by previous bioinformatic, biochemical and structural studies.

Keywords: Haloferax volcanii, archaea, Haloarchaea, MCM helicase, DNA replication, reverse genetics, zinc binding domain

\section{INTRODUCTION}

In all forms of life, successful chromosomal DNA replication requires efficient unwinding of the DNA double helix at the replication forks, a reaction catalyzed by the replicative DNA helicase. In eukaryotes the replicative helicase is the CMG complex, a tripartite molecular machine composed of Cdc45, MCM and GINS (reviewed by Onesti and MacNeill, 2013). The MCM (mini-chromosome maintenance) complex is the catalytic core of this machine (Vijayraghavan and Schwacha, 2012). MCM is a ring-shaped hexamer composed of six related but non-identical subunits, each of which is a member of the AAA+ (ATPases associated with diverse cellular activities) protein superfamily (Duderstadt and Berger, 2008). MCM is loaded onto chromosomal replication origins as a head-to-head double hexamer in the G1 phase of the cell cycle in a reaction known as replication licensing (Evrin et al., 2009; Remus et al., 2009; Gambus et al., 2011). Cdc45 and GINS then assemble at the G1-S boundary to form the CMG, activation of which involves MCM subunit phosphorylation by DDK (Dbf4-dependent protein kinase). Once activated, individual CMG complexes move with the replication forks from origin to inter-origin sequences.

Consistent with their shared evolutionary history, homologs of the major eukaryotic replication factors have been identified and characterized in the archaea, including homologs of the three components of the CMG. Owing to their high sequence similarity to their eukaryotic counterparts, archaeal MCM proteins were the first to be identified and biochemically characterized. Many archaea encode single MCM proteins that have been shown to form-or are presumed to form-homohexameric helicase complexes (reviewed by Slaymaker and Chen, 2012). The best studied examples of this type of MCM are from the euryarchaeon Methanothermobacter thermoautotrophicum and the crenarchaeon Sulfolobus solfataricus. However, a number of species encode multiple MCM proteins, such as Thermococcus kodakarensis and Methanococcus maripaludis, which encode three and four, respectively (Walters and Chong, 2010; Ishino et al., 
2011; Pan et al., 2011). It is not impossible that in some species these proteins could form eukaryotic-like heterohexameric complexes in vivo. Interestingly, only one of the three T. kodakarensis MCM proteins is essential for cell viability (Ishino et al., 2011; Pan et al., 2011)

Structural information is available for a number of archaeal MCM proteins and has been used to guide biochemical investigations of protein structure-function relationships (reviewed by Slaymaker and Chen, 2012). At a structural level, individual archaeal MCM proteins comprise a non-catalytic Nterminal domain followed by the catalytic AAA+ domain and, at the extreme C-terminus, a short winged helix-turnedhelix (wHTH) domain. The most extensive crystal structure is that of near full-length $S$. solfataricus MCM which spans the N-terminal and catalytic AAA+ domains but not the wHTH domain (Brewster et al., 2008). Efforts to determine the structure of the wHTH by NMR are ongoing (Wiedemann et al., 2013). Additional crystal structures include the $\mathrm{N}$ terminal domains of $S$. solfataricus, $M$. thermoautotrophicum, and Thermoplasma acidophilum MCM proteins, with the latter forming a right-handed spiral filament (Fletcher et al., 2003; Liu et al., 2008; Fu et al., 2014). A left-handed filament structure for near full-length $S$. solfataricus MCM has also been determined (Slaymaker et al., 2013), as well as a full-length structure of a catalytically inactive MCM homolog from Methanopyrus kandleri (Bae et al., 2009). The biological significance, if any, of the filamentous forms remains to be determined.

Unlike the MCM proteins, archaeal GINS and Cdc45 homologs share only very limited sequence similarity with their eukaryotic counterparts (Marinsek et al., 2006). The eukaryotic GINS complex is a heterotetramer, comprising the related Sld5, Psf1, Psf2, and Psf3 proteins (reviewed by Kamada, 2012). Both homotetrameric and heterotetrameric (i.e., dimer of dimer or $\mathrm{A}_{2} \mathrm{~B}_{2}$ ) complexes have been identified in archaea and the structure of the T. kodakarensis $\mathrm{A}_{2} \mathrm{~B}_{2}$ heterotetrameric GINS has been solved (Oyama et al., 2011). Archaeal Cdc45 homologs have only very recently been positively identified as such (Sanchez-Pulido and Ponting, 2011; Krastanova et al., 2012; Makarova et al., 2012). These proteins belong to the RecJ nuclease branch of the DHH hydrolase superfamily. Unlike eukaryotic Cdc45 proteins, at least some archaeal RecJ/Cdc45 proteins possess nuclease activity (Li et al., 2011; Yuan et al., 2013), the precise function of which is unclear. The existence of all three CMG components in archaea suggest that these organisms may have a valuable role to play as models for dissecting the function of the individual CMG components.

Using multiple sequence alignments and crystal structures as a guide, a number of laboratories have reported detailed mutagenesis studies of MCM structure-function relationships (reviewed by Slaymaker and Chen, 2012). In all cases, the effects of the mutations on MCM function were determined in vitro, using purified recombinant proteins in various biochemical assays. To our knowledge, there has been no in vivo reverse genetic analysis of the effects of mutations of MCM function, largely due to the difficulty or impossibility of conducting such studies in species where genetic tools are either rudimentary or unavailable. In this report we describe the first results of reverse genetic analysis of archaeal MCM function in vivo, using the haloarchaeal organism Haloferax volcanii as a model system. The haloarchaea present a particularly attractive model to study archaeal chromosome replication owing to the ease with which representative species can be manipulated genetically (reviewed by Farkas et al., 2013). Hfx. volcanii in particular has proved a highly successful model, with a number of components of the $H f x$. volcanii replication machinery already characterized, including multiple origins of replication (Hawkins et al., 2013), origin binding proteins (Norais et al., 2007), singlestranded DNA binding proteins (Skowyra and MacNeill, 2012; Stroud et al., 2012), the sliding clamp PCNA (Morgunova et al., 2009; Winter et al., 2009) and both ATP- and NAD-dependent DNA ligases (Poidevin and MacNeill, 2006; Zhao et al., 2006). In addition to their genetic tractability, over 100 haloarchaeal genomes have been now sequenced, offering a wealth of information for comparative protein sequence analysis. $H f x$. volcanii encodes a single MCM protein, a member of the previously defined core group of haloarchaeal MCM proteins discussed further below (MacNeill, 2009). In the work presented here, three conserved features of the protein are targeted for mutagenesis: the $\beta 7-\beta 8$ and $\beta 9-\beta 10 \beta$-hairpin loops and the four conserved cysteines of the putative zinc binding domain. The results of these studies establish $H f x$. volcanii as a valuable model for detailed structure-function analysis of MCM helicase and provide confirmation of the importance of these conserved features for MCM function in vivo.

\section{MATERIALS AND METHODS DATABASE SEARCHING AND SEQUENCE HANDLING}

Protein sequences were obtained from the UniProt Knowledgebase (UniProtKB) database (Magrane and UniProt Consortium, 2011): primary accession numbers are listed in Table 1. Multiple sequence alignments and phylogenetic trees were generated using ClustalX 2.1 (Larkin et al., 2007) and njplot (Perriere and Gouy, 1996), respectively. Intein sequences were initially identified by visual inspection as large sequence insertions in comparative sequence analysis. Inteins boundaries were defined by the presence of the N-terminal (block A) and C-terminal (block G) intein splicing motifs as defined at the InBase intein database (Perler, 2002).

\section{STRAINS AND GROWTH CONDITIONS}

Hfx. volcanii strains used in this study are listed in Table 2. All strains were grown in either $\mathrm{Hv}-\mathrm{YPC}$ or $\mathrm{Hv}-\mathrm{CA}$ medium at $45^{\circ} \mathrm{C}$ as described in the Halohandbook v7.2 (www.haloarchaea. com/resources/halohandbook). For selection procedures, tryptophan was added to $\mathrm{Hv}$-CA medium at a final concentration of $50 \mu \mathrm{g} / \mathrm{ml}$. For counter-selection using 5-fluoroorotic acid (5FOA), Hv-CA was supplemented with uracil and 5-FOA at final concentrations of $10 \mu \mathrm{g} / \mathrm{ml}$ and $50 \mu \mathrm{g} / \mathrm{ml}$, respectively. For mitomycin C sensitivity assays, wild-type (H26) and mutant cells were grown at $45^{\circ} \mathrm{C}$ in $\mathrm{Hv}$-YPC medium to an $\mathrm{OD}_{650 \mathrm{~nm}}$ of 0.2 0.32 , before being serially diluted in $18 \%$ SW. $5 \mu \mathrm{l}$ aliquots were then spotted on Hv-YPC plates containing 0, 10, 20, or $30 \mathrm{ng} / \mathrm{ml}$ mitomycin $\mathrm{C}$ and incubated at $45^{\circ} \mathrm{C}$ for 5 days. 
Table 1 | List of MCM proteins analyzed in this study.

\begin{tabular}{|c|c|c|c|c|c|c|c|}
\hline Key to Figure 1 & Species & UniProtKB accession number & Length (amino acids) & \multicolumn{4}{|c|}{ Inteins } \\
\hline 1 & Haladaptatus paucihalophilus DX253 & E7QNU9 & 698 & & & & \\
\hline 2 & Halalkalicoccus jeotgali B3 & D8J3U5 & 700 & & & & \\
\hline $3 \mathrm{~A}$ & Haloarcula marismortui ATCC 43049 & Q5UYX8 & 1175 & + & & & \\
\hline 3B & & Q5V011 & 681 & & & & \\
\hline $5 \mathrm{~A}$ & Halobiforma lacisalsi AJ5 & MoLR18 & 1342 & & & + & \\
\hline $5 B$ & & MoLZ47 & 312 & & & & \\
\hline 6 & Halococcus thailandensis JCM 13552 & MON8X7 & 698 & & & & \\
\hline 7 & Haloferax volcanii DS2 & D4GZG5 & 702 & & & & \\
\hline 8 & Halogeometricum borinquense DSM 11551 & E4NRK9 & 1818 & + & & + & \\
\hline 11B & & F8DEM3 & 698 & & & & \\
\hline $11 \mathrm{C}$ & & F8DET6 & 315 & & & & \\
\hline 12 & Haloquadratum walsbyi HBSQ001 & Q18E84 & 2216 & + & + & + & + \\
\hline 13 & Halorhabdus utahensis AX-2 & C7NUH7 & 1412 & + & & + & \\
\hline $14 \mathrm{~A}$ & Halorubrum lacusprofundi DSM 5036 & B9LTB1 & 700 & & & & \\
\hline $14 \mathrm{~B}$ & & B9LUI3 & 717 & & & & \\
\hline 15 & Halosarcina pallida JCM 14848 & MoD6So & 1172 & + & & & \\
\hline $16 \mathrm{~A}$ & Halosimplex carlsbadense 2-9-1 & MoD2C8 & 698 & & & & \\
\hline $16 \mathrm{~B}$ & & MOCE93 & 712 & & & & \\
\hline $17 \mathrm{~A}$ & Haloterrigena turkmenica DSM 5511 & D2RUS4 & 700 & & & & \\
\hline 17B & & D2S3H9 & 314 & & & & \\
\hline 23 & Natronolimnobius innermongolicus JCM 12255 & L9X2E8 & 700 & & & & \\
\hline $24 \mathrm{~A}$ & Natronomonas pharaonis DSM 2160 & Q3IML4 & 1037 & + & & & \\
\hline $24 \mathrm{~B}$ & & Q3IPB6 & 676 & & & & \\
\hline 25 & Natronorubrum tibetense GA33 & L9VHE4 & 1814 & + & & + & \\
\hline \multirow[t]{14}{*}{26} & Salinarchaeum sp. Harcht-Bsk1 & R4W989 & 697 & & & & \\
\hline & Methanosarcina acetivorans $\mathrm{C} 2 \mathrm{~A}$ & Q8TSW4 & 701 & & & & \\
\hline & Methanothermobacter thermautotrophicus $\Delta \mathrm{H}$ & O27798 & 666 & & & & \\
\hline & Sulfolobus solfataricus P2 & Q9UXG1 & 686 & & & & \\
\hline & Cenarchaeum symbiosum A & AORYB8 & 697 & & & & \\
\hline & Korarchaeum cryptofilum OPF8 & B1L6L9 & 703 & & & & \\
\hline & Thermoplasma acidophilum DSM 1728 & Q9HK10 & 698 & & & & \\
\hline & Archaeoglobus fulgidus DSM 4304 & O29733 & 586 & & & & \\
\hline & Homo sapiens $\mathrm{Mcm} 2$ & P49736 & 904 & & & & \\
\hline & Homo sapiens $\mathrm{Mcm} 3$ & P25205 & 808 & & & & \\
\hline & Homo sapiens Mcm4 & P33991 & 863 & & & & \\
\hline & Homo sapiens Mcm5 & P33992 & 734 & & & & \\
\hline & Homo sapiens Mcm6 & Q14566 & 821 & & & & \\
\hline & Homo sapiens $\mathrm{Mcm} 7$ & P33993 & 719 & & & & \\
\hline
\end{tabular}

The numbers shown in the first column provide a key for the proteins represented in the phylogenetic tree shown in Figure 1. The plus signs in the final four columns indicate the present of intein insertions at each of the four conserved positions A-D shown in Figure 2. Protein 3A, for example, Q5UYX8 from Haloarcula marismortui, has a single intein at position $A$ 
Table 2 | Haloferax volcanii strains used in this study.

\begin{tabular}{|c|c|c|c|}
\hline Strain no. & Genotype & Notes & References \\
\hline SMH693 & - & Wild-type strain DS70 & $\begin{array}{l}\text { Wendoloski } \\
\text { et al., } 2001\end{array}$ \\
\hline SMH656 & mcm-rM1 $\Delta$ pyrE2 & Single alanine substitution Q186A & This study \\
\hline SMH654 & mcm-rM3 $\triangle p y r E 2$ & Single alanine substitution E190A & This study \\
\hline SMH662 & mcm-rM6 $\triangle$ pyrE2 & Single alanine substitution Q199A & This study \\
\hline SMH638 & $m c m-b H 1 \Delta p y r E 2$ & Paired alanine substitution H226A/I227A & This study \\
\hline $\mathrm{SMH} 640$ & $m c m-b H 2 \Delta p y r E 2$ & Paired alanine substitution E228A/Q229A & This study \\
\hline SMH642 & $m c m-b H 3 \Delta p y r E 2$ & Paired alanine substitution $\mathrm{Q} 230 \mathrm{~A} / \mathrm{T} 231 \mathrm{~A}$ & This study \\
\hline $\mathrm{SMH} 644$ & $m c m-b H 4 \Delta p y r E 2$ & Paired alanine substitution S232A/G233A & This study \\
\hline SMH652 & mom-S1 $\triangle$ pyrE2 & Silent Acc65I restriction site replacing codons 141 and 142 & This study \\
\hline
\end{tabular}

\section{MOLECULAR CLONING REAGENTS}

Enzymes for molecular cloning were purchased from New England Biolabs (NEB), Promega or Fermentas. Oligonucleotides were synthesized by DNA Technology A/S (Risskov, Denmark). DNA sequencing was performed by Eurofins MWG Operon (Ebersberg, Germany). DNA purification kits were from Qiagen. PCR amplification was performed using the GC-rich PCR system (Roche) with Taq polymerase (NEB) substituting for the GC-rich enzyme as necessary. For routine cloning purposes, E.coli DH5 $\alpha$ (fhuA2 $\Delta$ (argF-lacZ)U169 phoA glnV44 $880 \Delta$ (lacZ)M15 gyrA96 recA1 relA1 endA1 thi-1 hsdR17) was used (Invitrogen). To prepare unmethylated plasmid DNA for $H f x$. volcanii transformation, E.coli GM121 (F- dam-3 dcm-6 ara-14 fhuA31 galK2 galT22 hdsR3 lacY1 leu-6 thi-1 thr-1 tsx-78) was used.

\section{CONSTRUCTION OF MUTANT Hfx. Volcanii STRAINS}

Mutant strains were constructed using the pop-in/pop-out method (Bitan-Banin et al., 2003) in Hfx. volcanii $\Delta$ pyrE2 strain H26 as follows. First, plasmid pTA131-HfxMCM-HXba was constructed by using oligonucleotide primers HfxMCM-5H and HfxMCM-3X (designed to include HindIII and XbaI sites respectively, see Table 3 ) to amplify a $1.0 \mathrm{~kb}$ fragment of $H f x$. volcanii genomic DNA spanning the region from 100 nucleotides upstream of the $\mathrm{mcm}$ ORF to 900 nucleotides inside the ORF. The PCR product was digested with HindIII and XbaI, cloned into plasmid pTA131 digested with the same two enzymes (Allers et al., 2004) and sequenced to confirm the absence of PCR errors.

The resulting plasmid (pTA131-HfxMCM-HXba) was then used as a template for PCR overlap extension mutagenesis (OEM) to create 25 mutant derivatives. The sequences of the mutagenic primers are given in Table 3. OEM was performed using the GC-Rich PCR system buffer and GC-Rich enzyme mix (Roche) for the first round of PCR and the GC-Rich PCR system buffer and Taq polymerase (NEB) for the second round. Oligonucleotides HfxMCM-5H and HfxMCM-3X were used as the flanking primers throughout. The resulting PCR products were then restricted with Bsu36I and BstEII (for $\beta 7-\beta 8 \beta$-hairpin and zinc binding domain mutants) or BstEII and XbaI (for $\beta 9$ $\beta 10 \beta$-hairpin mutants) and the 329 bp Bsu36I-BstEII or 271 bp BstEII-XbaI pieces carrying the mutations cloned back into pTA131-HfxMCM-HXba from which the corresponding wildtype restriction fragments had been removed. Plasmids were again sequenced to confirm the absence of unwanted sequence changes before being passaged through E.coli GM121 to generate unmethylated DNA for transformation into $H f x$. volcanii $\triangle p y r E 2$ strain H26 (Table 2).

Transformation of $H f x$.volcanii was accomplished as described in the Halohandbook v7.2 (www.haloarchaea.com/resources/ halohandbook). Transformants obtained on Hv-CA medium lacking uracil were grown for 30 generations at $45^{\circ} \mathrm{C}$ in nonselective $\mathrm{Hv}-\mathrm{YPC}$ medium to allow loss of the plasmid before being plated on $\mathrm{Hv}-\mathrm{CA}$ plates containing $50 \mu \mathrm{g} / \mathrm{ml} 5$-FOA and a $10 \mu \mathrm{g} / \mathrm{ml}$ uracil. Colonies formed on these plates were then inoculated into $500 \mu \mathrm{l}$ of $\mathrm{Hv}$-YPC liquid medium and grown overnight at $45^{\circ} \mathrm{C}$. Genomic DNA from these was prepared by taking $10 \mu \mathrm{l}$ of overnight culture, adding to $500 \mu \mathrm{l}$ of sterile water and heating to $70^{\circ} \mathrm{C}$ for $10 \mathrm{~min}$ to lyse the cells. For the $\beta$-hairpin and zinc binding domain mutants, $1 \mu \mathrm{l}$ of the resulting mix was used in PCR reactions to screen for the presence of the mutants in the chromosome. To discriminate between wild-type and mutant sequences, oligonucleotide primers with mismatched $3^{\prime}$ sequences were used in PCR reactions in conjunction with primer HFXMCM-R1150, which lies 1150 nucleotides into the $\mathrm{mcm}$ ORF and which is therefore not present in pTA131-HfxMCM-HXba (see Table 3 for oligonucleotide sequences). For the silent restriction site mutant, the partial $\mathrm{mcm}$ ORF was amplified using primers HfxMCM-5H and HfxMCM-3X and the PCR products digested with Acc65I to identify mutants. In all cases, putative positive clones were then re-streaked twice to single colonies on Hv-YPC agar at 
Table 3 | Oligonucleotides used in this study.

\begin{tabular}{|c|c|}
\hline Primer name & Sequence \\
\hline \multicolumn{2}{|c|}{ A. FOR CONSTRUCTION OF pTA131-HfxMCM-HXba } \\
\hline HfхMCM-5H & 5'-GTGTGTGTGTAAGCTTCCTCCGCGAGGCGACGGA-3' \\
\hline HfхMCM-3X & 5'-GGTGGTGGTGTCTAGACATGGCAATCTTCTCCTG-3' \\
\hline
\end{tabular}

\section{B. FOR CONSTRUCTION OF $\beta$ 7- $\beta 8$ $\beta$-HAIRPIN MUTANTS BY OEM}

HFXMCM-rM1-F

HFXMCM-rM1-R

5'-AAACTGCGCGTCGCCGAGTCCCCCGAGGGC-3'

HFXMCM-rM2-F

HFXMCM-rM2-R

HFXMCM-rM3-F

HFXMCM-rM3-R

HFXMCM-rM4-F

HFXMCM-rM4-R

HFXMCM-rM5-F

HFXMCM-rM5-R

HFXMCM-rM6-F

HFXMCM-rM6-R

HFXMCM-rD1-F

HFXMCM-rD1-R

HFXMCM-rD2-F

HFXMCM-rD2-R

HFXMCM-rD3-F

HFXMCM-rD3-R

HFXMCM-rD4-F

HFXMCM-rD4-R

5'-GCCCTCGGGGGACTCGGCGACGCGCAGTTT-3'

5'-AAACTGCGCGTCCAGGCCTCCCCCGAGGGCCTGCG-3'

5'-CGCAGGCCCTCGGGGGAGGCCTGGACGCGCAGTTT-3'

5'-CAGGAGTCCCCCGCCGGCCTGCGCGGGGGC-3'

5'-GCCCCCGCGCAGGCCGGCGGGGGACTCCTG-3'

5'-CCCGAGGGCCTGGCGGGGGGCGAGACGCCG-3'

5'-CGGCGTCTCGCCCCCCGCCAGGCCCTCGGG-3'

5'-CTGCGCGGGGGCGCCACGCCGCAGAGCATC-3'

5'-GATGCTCTGCGGCGTGGCGCCCCCGCGCAG-3'

5'-GGCGAGACGCCGGCCAGCATCGACATCAAC-3'

5'-GTTGATGTCGATGCTGGCCGGCGTCTCGCC-3'

5'-CAGGAGTCCCCCGAG-GGCGAGACGCCGCAG-3'

5'-CTGCGGCGTCTCGCC-CTCGGGGGACTCCTG-3'

5'-GTCCAGGAGTCCCCC-GAGACGCCGCAGAGC-3'

5'-GCTCTGCGGCGTCTC-GGGGGACTCCTGGAC-3'

5'-CGCGTCCAGGAGTCC-ACGCCGCAGAGCATC-3'

5'-GATGCTCTGCGGCGT-GGACTCCTGGACGCG-3'

5'-CTGCGCGTCCAGGAG-CCGCAGAGCATCGAC-3' 5'-GTCGATGCTCTGCGG-CTCCTGGACGCGCAG-3'

\section{FOR CONSTRUCTION OF $\beta$ 9- $\beta 10 \beta$-HAIRPIN MUTANTS BY OEM}

HFXMCM-bH1-F

HFXMCM-bH1-R

HFXMCM-bH2-F

HFXMCM-bH2-R

HFXMCM-bH3-F

HFXMCM-bH3-R

HFXMCM-bH4-F

HFXMCM-bH4-R

HFXMCM-bH5-F

HFXMCM-bH5-R

HFXMCM-bH6-F

HFXMCM-bH6-R

HFXMCM-hD1-F

HFXMCM-hD1-R

HFXMCM-hD2-F

HFXMCM-hD2-R

HFXMCM-hD3-F

HFXMCM-hD3-R

HFXMCM-hD4-F

HFXMCM-hD4-R

5'-GTCGGCGTCCTCGCAGCGGAACAGCAGACATCG-3'

5'-CGATGTCTGCTGTTCCGCTGCGAGGACGCCGAC-3'

5'-GTCCTCCACATCGCAGCGCAGACATCGGGCAAC-3' 5'-GTTGCCCGATGTCTGCGCTGCGATGTGGAGGAC-3'

5'-CACATCGAACAGGCAGCGTCGGGCAACGAGAAG-3'

5'-CTTCTCGTTGCCCGACGCTGCCTGTTCGATGTG-3'

5'-GAACAGCAGACAGCAGCGAACGAGAAGACGCCC-3'

5'-GGGCGTCTTCTCGTTCGCTGCTGTCTGCTGTTC-3'

5'-CAGACATCGGGCGCAGCGAAGACGCCCGTCTTC-3'

5'-GAAGACGGGCGTCTTCGCTGCGCCCGATGTCTG-3'

5'-TCGGGCAACGAGGCAGCGCCCGTCTTCGACTAC-3'

5'-GTAGTCGAAGACGGGCGCTGCCTCGTTGCCCGA-3'

5'-CACATCGAACAGCAG-GGCAACGAGAAGACG-3'

5'-CGTCTTCTCGTTGCC-CTGCTGTTCGATGTG-3'

5'-CTCCACATCGAACAG-AACGAGAAGACGCCC-3'

5'-GGGCGTCTTCTCGTT-CTGTTCGATGTGGAG-3'

5'-GTCCTCCACATCGAA-GAGAAGACGCCCGTC-3'

5'-GACGGGCGTCTTCTC-TTCGATGTGGAGGAC-3'

5'-GGCGTCCTCCACATC-AAGACGCCCGTCTTC-3'

5'-GAAGACGGGCGTCTT-GATGTGGAGGACGCC-3'

\section{FOR CONSTRUCTION OF ZINC BINDING DOMAIN MUTANTS BY OEM}

HFXMCM-C137A-F 5'-CCGCCTTCGAGGCGCAGCGCTGCGG-3'

HFXMCM-C137A-R 5'-CCGCAGCGCTGCGCCTCGAAGGCGG-3'

HFXMCM-C140A-F 5'-AGTGCCAGCGCGCGGGGACGATGAG-3'

HFXMCM-C140A-R 5'-CTCATCGTCCCCGCGCGCTGGCACT-3'

HFXMCM-C159A-F 5'-AACCCCACGAGGCGCAGGGATGCGA-3'5'-

HFXMCM-C159A-R TCGCATCCCTGCGCCTCGTGGGGTT-3'

HFXMCM-C162A-F 5'-AGTGTCAGGGAGCGGAGCGCCAGGG-3'

HFXMCM-C162A-R 5'-CCCTGGCGCTCCGCTCCCTGACACT-3'

\section{E. FOR CONSTRUCTION OF SILENT Acc65I mutation by OEM}

HFXMCM-S1-F

5'-TGCCAGCGCTGCGGTACCATGAGCTACATC-3'

HFXMCM-S1-R
Table 3 | Continued

\begin{tabular}{ll}
\hline Primer name & \multicolumn{1}{c}{ Sequence } \\
\hline F. FOR DETECTION OF $\boldsymbol{\beta}$ 7- $\beta 8$ $\boldsymbol{\beta}$-HAIRPIN MUTANTS \\
HFXMCM-rM1-WT & 5'-CAGAAACTGCGCGTCCAG-3' \\
HFXMCM-rM1-MUT & 5'-CAGAAACTGCGCGTCGCC-3' \\
HFXMCM-rM2-WT & 5'-AAACTGCGCGTCCAGGAG-3' \\
HFXMCM-rM2-MUT & 5'-AAACTGCGCGTCCAGGCC-3' \\
HFXMCM-rM3-WT & 5'-GTCCAGGAGTCCCCCGAG-3' \\
HFXMCM-rM3-MUT & 5'-GTCCAGGAGTCCCCCGCC-3' \\
HFXMCM-rM4-WT & 5'-TCCCCCGAGGGCCTGCGC-3' \\
HFXMCM-rM4-MUT & 5'-TCCCCCGAGGGCCTGGCG-3' \\
HFXMCM-rM5-WT & 5'-GGCCTGCGCGGGGGCGAG-3' \\
HFXMCM-rM5-MUT & 5'-GGCCTGCGCGGGGGCGCC-3' \\
HFXMCM-rM6-WT & 5'-GGGGGCGAGACGCCGCAG-3' \\
HFXMCM-rM6-MUT & 5'-GGGGGCGAGACGCCGGCC-3' \\
HFXMCM-rD1-MUT & 5'-GGAGTCCCCCGAGGGCGAG-3' \\
HFXMCM-rD2-MUT & 5'-CCAGGAGTCCCCCGAGAC-3' \\
HFXMCM-rD3-MUT & 5'-CGTCCAGGAGTCCACGCC-3' \\
HFXMCM-rD4-MUT & 5'-CTGCGCGTCCAGGAGCCG-3'
\end{tabular}

\section{G. FOR DETECTION OF $\beta$ 9- $\beta 10 \beta$-HAIRPIN MUTANTS}

HFXMCM-bH1-WT 5'-GTCGGCGTCCTCCACATC-3'

HFXMCM-bH1-MUT 5'-GTCGGCGTCCTCGCAGCG-3'

HFXMCM-bH2-WT 5'-GTCCTCCACATCGAACAG-3'

HFXMCM-bH2-MUT 5'-GTCCTCCACATCGCAGCG-3'

HFXMCM-bH3-WT 5'-CACATCGAACAGCAGACA-3'

HFXMCM-bH3-MUT 5'-CACATCGAACAGGCAGCG-3'

HFXMCM-bH4-WT 5'-GAACAGCAGACATCGGGC-3'

HFXMCM-bH4-MUT 5'-GAACAGCAGACAGCAGCG-3'

HFXMCM-bH5-WT 5'-CAGACATCGGGCAACGAG-3'

HFXMCM-bH5-MUT 5'-CAGACATCGGGCGCAGCG-3'

HFXMCM-bH6-WT 5' 5 -ACATCGGGCAACGAGAAGA-3'

HFXMCM-bH6-MUT 5'-ACATCGGGCAACGAGGCAG-3'

HFXMCM-hD1-WT 5'-CACATCGAACAGCAGACA-3'

HFXMCM-hD1-MUT 5'-CACATCGAACAGCAGGGC-3'

HFXMCM-hD2-WT 5'-CTCCACATCGAACAGCAG-3'

HFXMCM-hD2-MUT 5'-CTCCACATCGAACAGAAC-3'

HFXMCM-hD3-WT 5'-TCCTCCACATCGAACAGC-3'

HFXMCM-hD3-MUT 5' 5 -TCCTCCACATCGAAGAGA-3'

HFXMCM-hD4-WT 5'-GGCGTCCTCCACATCGAA-3'

HFXMCM-hD4-MUT 5'-GGCGTCCTCCACATCAAG-3'

H. FOR DETECTION OF ZINC BINDING DOMAIN MUTANTS

HFXMCM-C137A-WT 5'-GAAGCCGCCTTCGAGTGC-3'

HFXMCM-C137A-MUT 5'-GAAGCCGCCTTCGAGGCG-3'

HFXMCM-C140A-WT 5'-TTCGAGTGCCAGCGCTGC-3'

HFXMCM-C140A-MUT 5'-TTCGAGTGCCAGCGCGCG-3'

HFXMCM-C159A-WT 5'-CAGGAACCCCACGAGTGT-3'

HFXMCM-C159A-MUT 5'-CAGGAACCCCACGAGGCG-3'

HFXMCM-C162A-WT 5'-CACGAGTGTCAGGGATGC-3'

HFXMCM-C162A-MUT 5'-CACGAGTGTCAGGGAGCG-3'

\section{REVERSE PRIMER FOR MUTANT DETECTION}

HFXMCM-R1150 5'-GCGAATCCGCGAGCCGTC-3'

Restriction sites in oligonucleotide primers used for PTA131-HfXMCM-HXba construction underlined. Oligonucleotide primers for overlap extension mutagenesis (OEM) are shown in top strand-bottom strand pairs with the mutated bases underlined in the top strand primer (for amino acid substitutions) or with deletion boundaries indicated with a dash. Oligonucleotide primers for mutant detection are shown with $3^{\prime}$ mismatched bases underlined. 
$45^{\circ} \mathrm{C}$ before being re-tested by PCR using wild-type and mutant primers. Finally, the presence of the mutation was confirmed by sequencing of the relevant part of the mcm ORF amplified by PCR from genomic DNA (see Supplementary Information for DNA sequence traces).

\section{RESULTS}

\section{HALOARCHAEAL MCM PROTEINS}

Prior to embarking on reverse genetic analysis of $H f x$. volcanii MCM protein function, we undertook a detailed bioinformatic analysis of MCM protein distribution and conservation across the haloarchaea. To identify haloarchaeal MCM proteins, we searched the UniProtKB sequence database using BLAST with the $H f x$. volcanii MCM protein (HVO_0220, UniProtKB accession number D4GZG5) as the query sequence. Almost 200 proteins were identified in $\sim 120$ species belonging to 26 different genera of the Halobacteriaceae. To simplify further analysis, we selected a single species as a representative of each genus. These 26 species encoded a total of $39 \mathrm{MCM}$ proteins. A complete list of the species under investigation, together with accession numbers for the proteins we identified, can be found in Table 1 .

To investigate the relationship between the proteins in greater detail, we constructed multiple protein sequence alignments using ClustalX 2.1 (Larkin et al., 2007). Previously, when analysing a significantly smaller number of genomes (five), we identified a core group of five MCM proteins and a further three proteins that we designated as outliers (MacNeill, 2009). We performed a similar analysis of the larger dataset, using ClustalX to generate multiple sequence alignments of protein sequences (from which inteins were first removed-see below) and njplot (Perriere and Gouy, 1996) to generate unrooted phylogenetic trees. Figure 1 shows the phylogenetic tree for the 39 proteins. As before, two groups are apparent, corresponding to the core and outlier groups defined previously (MacNeill, 2009). Each of the 26 species encodes a single member of the core group that includes the Hfx. volcanii MCM protein. The core group MCM proteins range in length from 697 to 702 amino acids (after inteins are removed-see below) and display a minimum pairwise protein sequence identity of $68 \%$ (range 68-95\%). Three hundred and twenty four residues ( $46 \%$ of the protein sequence) are absolutely conserved across all 26 core group proteins. This includes the key catalytic residues that make up the Walker A (P-loop) motif (GDPGTGKS in all 26 proteins compared to the consensus $\mathrm{GX}_{4} \mathrm{GKS} / \mathrm{T}$ ), the Walker B motif (DELD in all 26 proteins) and arginine finger motif (SRF in all 26 proteins).

The outlier group proteins display much greater variety in length (312-717 amino acids) and sequence similarity (24-48\% identity with $H f x$. volcanii MCM). The four shortest proteins (312-315 amino acids) are made up of sequences related to the non-catalytic N-terminal domain of MCM only and cannot possess helicase activity. The remaining nine outliers all possess conserved Walker B (DEL/ID) and arginine finger (SRF) motifs, as well as the key lysine in the Walker A (P-loop) motif, suggesting that these proteins may well have ATPase and/or helicase activities. To date, none has been characterized biochemically.

Inteins are parasitic genetic elements capable of efficient selfsplicing at the protein level (Gogarten and Hilario, 2006) and are

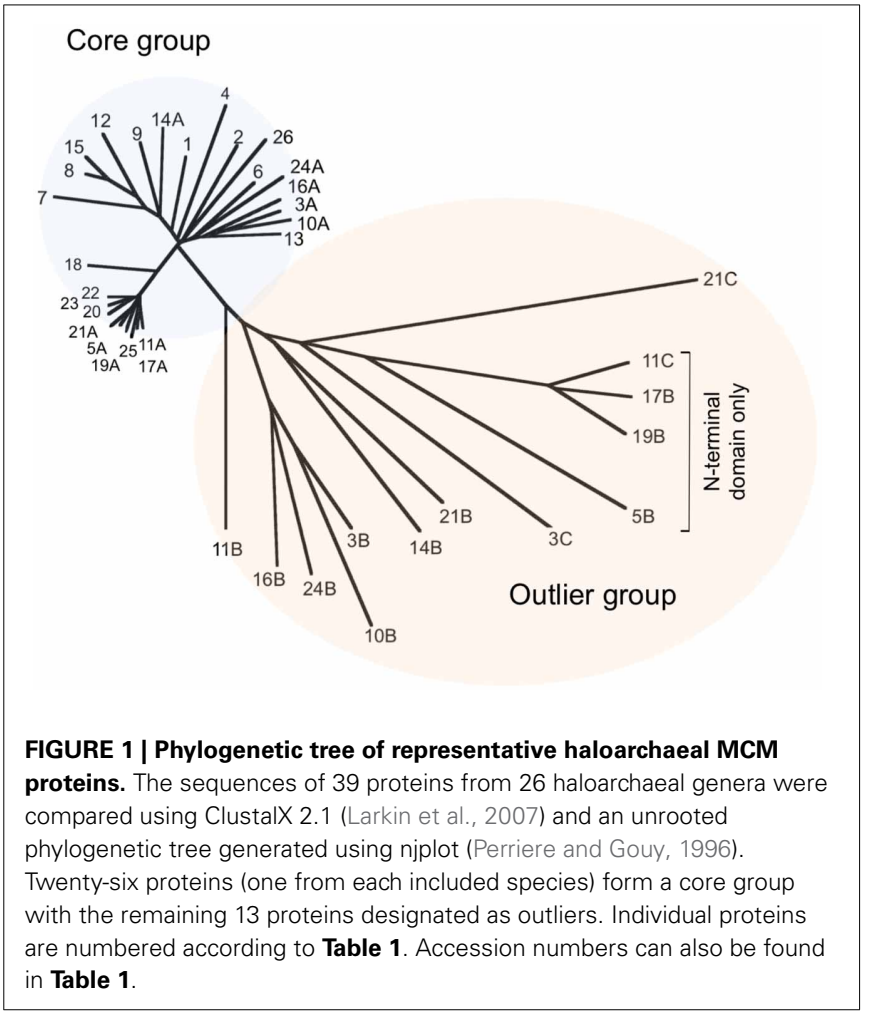

a common feature of the haloarchaeal MCM proteins. Amongst the 39 proteins, inteins were found in 13, all members of the core group defined above, and at four different locations within the C-terminal catalytic domain of the protein, with the largest number of inteins in an individual MCM protein being four (Table 1). Figure 2 shows the position of the four inteins relative to conserved sequence features found in archaeal MCM proteins. As noted previously, inteins are frequently located at or very near to highly conserved and functionally important sequence regions (Gogarten and Hilario, 2006) and the haloarchaeal MCM inteins are no exception: intein insertion site $\mathrm{A}$ is located immediately C-terminal to the essential lysine in the Walker A sequence GDPGTGKS mentioned above, while intein $\mathrm{C}$ lies just four amino acids C-terminal to the Walker B sequence DELD (Table 1, Figure 2). Intein splicing is therefore likely to be essential for protein function.

In a number of archaeal organisms, including representatives of the crenarchaea, euryarchaea, thaumarchaea and korarchaea, the gene encoding MCM is found adjacent to that encoding a GINS subunit (MacNeill, 2010). To ask whether this arrangement is also found in any of the species under investigation in this report, we examined the chromosomal context of each of the 26 core MCM genes and also the 13 outlier proteins. None of the genes encoding core group proteins is located adjacent to a gene encoding GINS or indeed, to any known replication gene (data not shown). A similar situation is seen with genes encoding 12 of the 13 outlier proteins, the sole exception being the pNG3053 protein (UniProtKB accession number Q5V814, labeled 3C in Figure 1) encoded by plasmid pNG300 of Haloarcula marismortui which is located immediately $3^{\prime}$ to an ORF encoding 


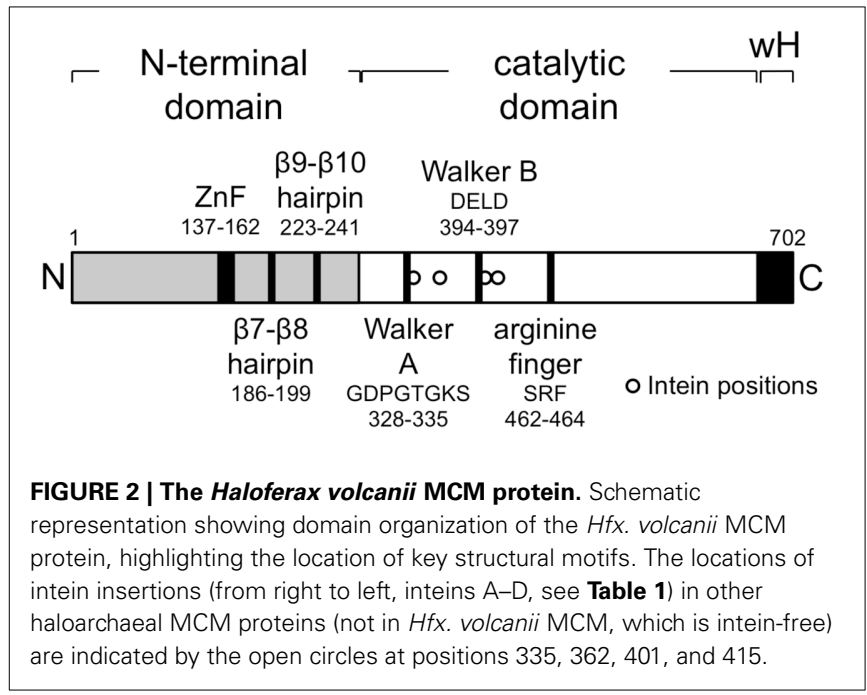

the C-terminally truncated GINS homolog pNG3052 (data not shown).

\section{REVERSE GENETIC ANALYSIS OF MCM FUNCTION}

Hfx. volcanii encodes a single intein-free MCM protein of 702 amino acids in length (Table $\mathbf{1}$ ) that comprises an $\mathrm{N}$-terminal domain that spans residues 1-283, an AAA+ catalytic core domain spanning residues 283-632 and a C-terminal winged helix-turn-helix (wHTH) domain spanning residues 633-702. All the conserved sequence features characteristic of archaeal MCM proteins are found in the $H f x$. volcanii protein (Figure 2). In order to test whether $H f x$. volcanii was a workable model for reverse genetic analysis of archaeal MCM function, three of these conserved features were targeted for mutagenesis: the $\beta 7-\beta 8 \beta$-hairpin loop (also known as the allosteric communication loop, ACL), the $\beta 9-\beta 10 \beta$-hairpin loop and the putative zinc binding domain (Figure 2).

To introduce mutations into the $\mathrm{mcm}$ gene, we used the popin/pop-out method (Bitan-Banin et al., 2003). To achieve this, a plasmid was constructed carrying a $1.0 \mathrm{~kb}$ region of the $H f x$. volcanii genome spanning the first 900 nucleotides of the $\mathrm{mcm}$ open reading frame together with 100 nucleotides of $5^{\prime}$ flanking region. The plasmid also carries the pyrE2 selectable marker (pyrE2 function is required for uracil prototrophy) but does not possess a replication origin capable of promoting autonomous replication in $H f x$. volcanii. Stable maintenance therefore requires that the plasmid integrates (pops-in) into the Hfx. volcanii genome by means of homologous recombination between the $\mathrm{mcm}$ sequences on the plasmid and the $\mathrm{mcm}$ gene in the chromosome. PCR overlap extension mutagenesis was used to generate a series of mutated forms of the plasmid in which the targeted amino acids were either replaced, singly or in pairs, with one or two alanine residues respectively, or deleted altogether. Next, the plasmids were introduced into $H f x$. volcanii $\triangle$ pyrE2 strain $\mathrm{H} 26$ by standard methods and transformant (popin) colonies obtained on Hv-CA plates lacking uracil. Multiple independent colonies were then individually picked and grown in non-selective (uracil-containing) Hv-YPC medium before being plated onto Hv-CA plates containing 5-fluoroorotic acid to select for (pop-out) clones that had lost the plasmid (see Materials and Methods). Pop-out colonies were then screened by PCR using oligonucleotide primers specific for either the wildtype or mutant sequences (see Table 3 for primer sequences). Candidate mutant strains were sequentially re-streaked twice on non-selective medium (Hv-YPC) before the presence of the mutation was confirmed by PCR using wild-type- and mutantspecific primers and by sequencing of amplified genomic DNA (see Materials and Methods). Table 2 lists the strains constructed by this method.

\section{MUTAGENESIS OF THE $\beta 7$ - $\beta 8 \beta$-HAIRPIN LOOP}

First identified on the basis of its high degree of sequence conservation across species, the $\beta 7-\beta 8 \beta$-hairpin loop (also known as the allosteric communication loop, ACL) is located at the interface between the $\mathrm{N}$-terminal and AAA + catalytic domains of the MCM protein (Figure 3). A number of mutants in $\beta 7-\beta 8$ loop have previously been analyzed biochemically, leading to the conclusion that the loop plays a role in coupling the activities of these two domains (Sakakibara et al., 2008; Barry et al., 2009). The mutations tested include six single point mutants in recombinant $M$. thermoautotrophicum MCM (including four at the boxed conserved residues shown in Figure 3B ) as well as a triple point mutant and a complete replacement of the loop by the tripeptide serine-asparagine-glycine in S. solfataricus MCM (Sakakibara et al., 2008; Barry et al., 2009).

In order to test whether the function of the $\beta 7-\beta 8$ loop function was essential in vivo in $H f x$. volcanii, we attempted to construct 10 different alleles (Figure 3): six single point mutants in which individual charged or bulky polar amino acids within the loop were replaced with alanine (mutants $\mathrm{mcm}-\mathrm{rM} 1-\mathrm{mcm}$ $r M 6)$ and four deletions, of two, four, six, and eight amino acids (mutants $m c m-r D 1-m c m-r D 4$, respectively). Two of the individual amino acids targeted (Q186 and E187) are conserved across species (Figure 3B); the equivalent residues were mutated in $M$. thermoautotrophicum MCM (Sakakibara et al., 2008).

Of the 10 mutants, five ( $m c m-r M 1, m c m-r M 3-m c m-r M 6)$ were readily identified by PCR screening using genomic DNA templates and oligonucleotide primers specific for the wildtype and mutant sequences (see Table 3 ) and confirmed by DNA sequencing (Supplementary Figure S1). Despite extensive screening (see Materials and methods), the remaining four strains ( $m c m-r M 2, m c m-r D 1-m c m-r D 4)$ could not be isolated, suggesting that these mutations either inactivate or significantly impair the function of the $H f x$. volcanii MCM protein in cells grown at $45^{\circ} \mathrm{C}$. Growth of the five viable mutants was indistinguishable from the parental wild-type strain $\mathrm{H} 26$ (Figure 4A, upper panel). These results indicate that the $\beta 7-\beta 8$ loop is indeed essential for $H f x$. volcanii MCM function although it is also clear that some point mutations (including replacement of conserved amino acid glutamine 186 with alanine) can be tolerated.

\section{MUTAGENESIS OF THE $\beta$ 9- $\beta 10 \beta$-HAIRPIN LOOP}

Structural analysis of archaeal MCM proteins identifies four $\beta$ hairpins in each monomer, three of which protrude, to a greater or lesser extent, into the central channel through which single- 


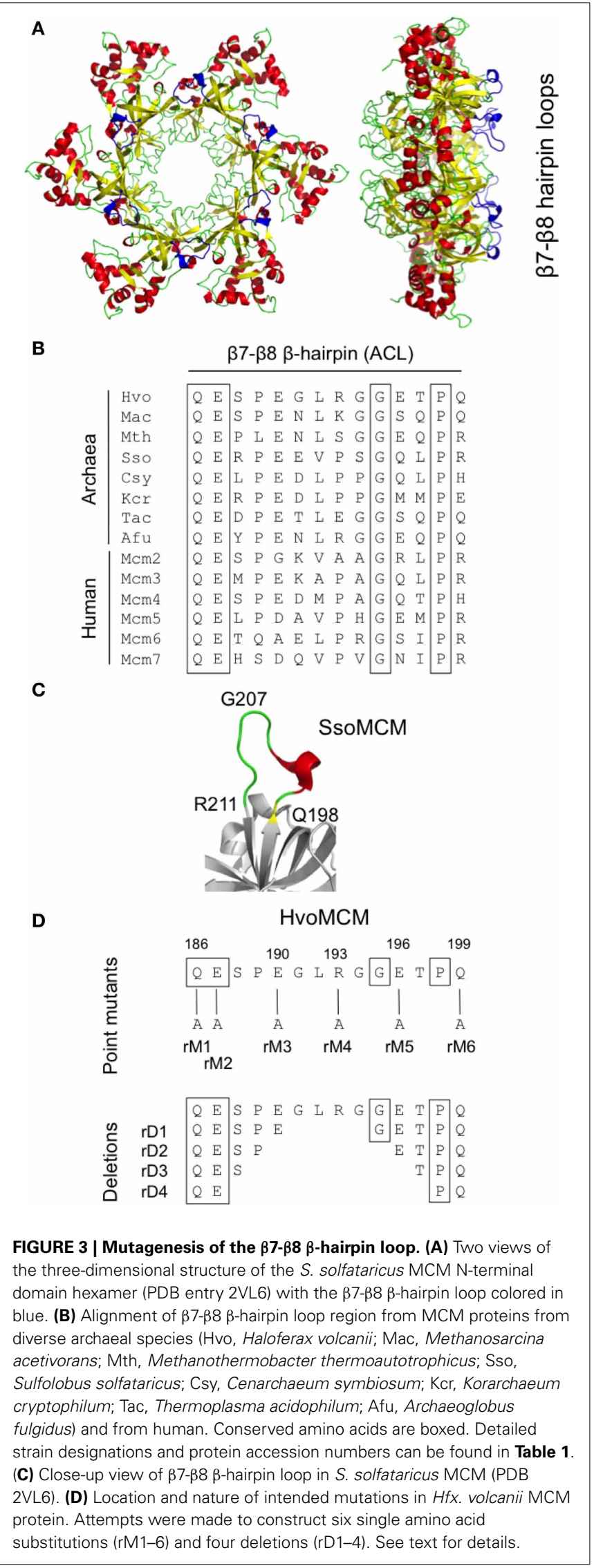

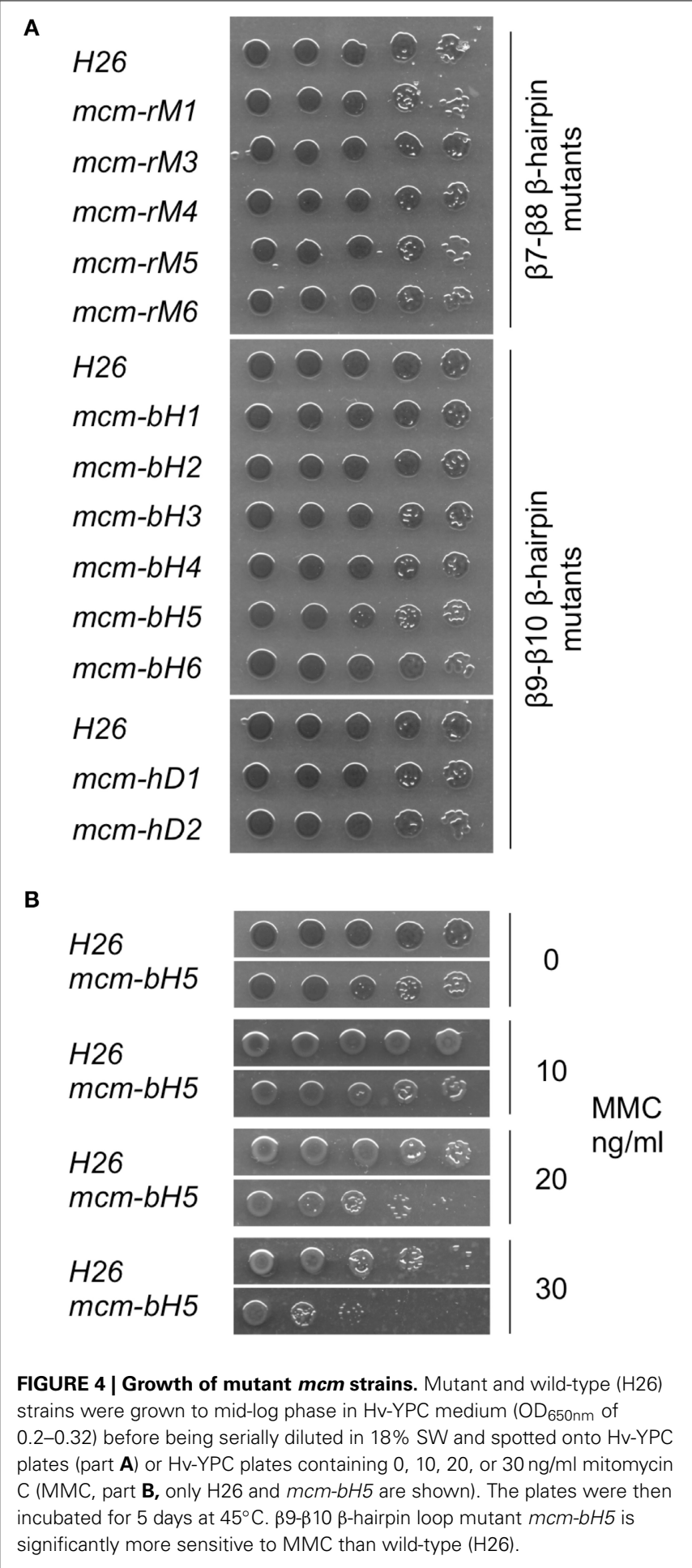

or double-stranded DNA is thought to pass (Slaymaker and Chen, 2012). One of these hairpins, the positively charged $\beta 9$ $\beta 10 \beta$-hairpin (also known as the NT-hairpin) is located in the $\mathrm{N}$-terminal domain of the protein (see Figure 5A). Unlike the $\beta 7-\beta 8$ loop described above, the sequence of this part of the MCM protein is not well-conserved across evolution (Figure 5B). 


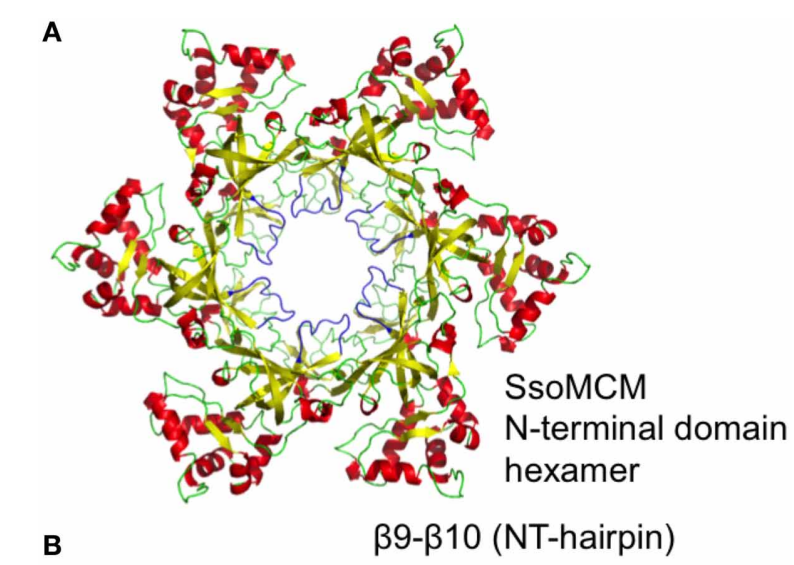

B

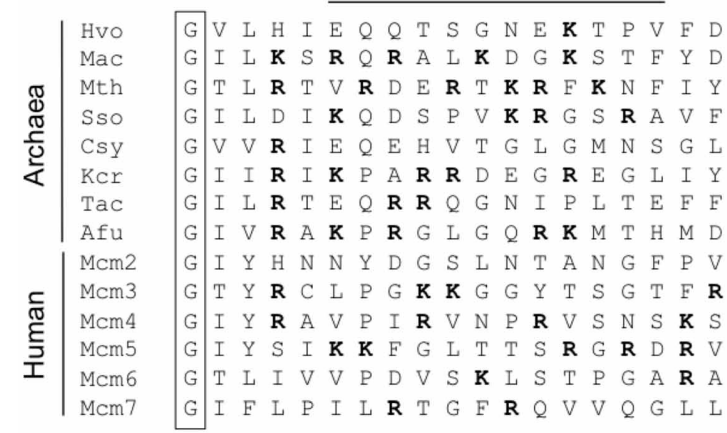

C

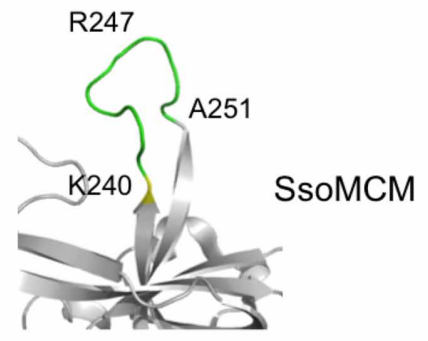

D
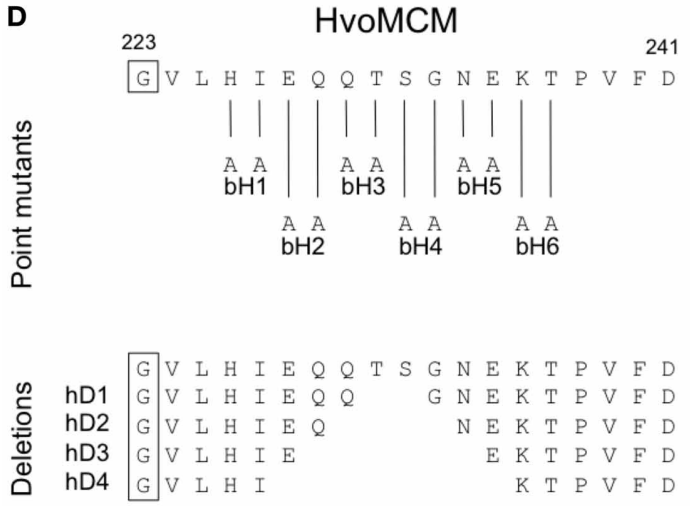

FIGURE 5 | Mutagenesis of the $\beta 9-\beta 10 \beta$-hairpin (NT-hairpin). (A) Structure of the $S$. solfataricus MCM N-terminal domain hexamer (PDB $2 \mathrm{VL6}$ ) with the $\beta 9-\beta 10 \beta$-hairpins highlighted in blue. (B) Multiple sequence alignment of the $\beta 9-\beta 10$-hairpin region from diverse archaeal species and from human (see legend to Figure $\mathbf{3}$ for key and Table $\mathbf{1}$ for strain details and protein accession numbers). Basic amino acids are highlighted in bold type. (C) Close-up of S. solfataricus MCM $\beta 9-\beta 10$ hairpin loop. (D) Location and nature of intended mutations in Hfx. volcanii MCM protein. Attempts were made to construct six paired alanine substitutions (bH1-6) and four short deletions (hD1-4). See text for details.
However, it appears that the positively charged nature of the hairpin is important for function: mutation of arginine 226 and lysine 228 in the M. thermoautotrophicum MCM abolishes that protein's ability to bind to DNA (Fletcher et al., 2003).

To probe the in vivo function of the $\beta 9-\beta 10 \beta$-hairpin loop in Hfx. volcanii, and in the absence of highly conserved amino acids presenting themselves as obvious targets for mutagenesis, we initially attempted to construct six mutants, mcm-bH1$m c m-b H 6$, in which adjacent amino acids were replaced with paired alanines (Figure 5D). All six mutants were recovered following PCR screening (and confirmed by sequencing, see Supplementary Figure S2), implying that the $\beta 9-\beta 10 \beta$-hairpin loop is readily mutable. None of six mutants exhibited obvious growth defects at $45^{\circ} \mathrm{C}$ (Figure $4 \mathrm{~A}$, middle panel).

We therefore extended this analysis by attempting to create strains carrying deletions of increasing size in the $\beta 9-\beta 10$ loop (mutants $m c m-h D 1-m c m-h D 4$, see Figure 5D). However, despite extensive screening, only two of the four could be isolated: $m c m-h D 1$ and $m c m-h D 2$. Growth of these strains, like $m c m-b H 1-m c m-b H 6$, was indistinguishable from the parental wild-type H26 (Figure 4A, lower panel). That we were unable to isolate $m c m-r D 3$ and $m c m-r D 4$ strains strongly implies that these deletions either inactivate or significantly impair the function of the Hfx. volcanii MCM protein. We conclude from this analysis that while the precise sequence of the $\beta 9-\beta 10 \beta$-hairpin loop is not absolutely required for the function of the protein, the loop itself does have a crucial role.

\section{MUTAGENESIS OF THE ZINC BINDING DOMAIN}

The N-terminal domain of the archaeal MCM proteins contains cysteine and histidine residues that fold into a zinc binding domain (Figure 6) (Slaymaker and Chen, 2012). A similar domain appears also to be present in eukaryotic MCM proteins but its precise function in either kingdom is unknown. We attempted to construct mutants in which each of the four cysteines was individually replaced with alanine (Figure 6C). However, none of these four mutants that we attempted to construct (mutants mcm-C137A, mcm-C140A, mcm-C159A, and $m c m-C 162 A$, see Figure 6C) could be isolated, implying that all four cysteines-and presumably zinc binding-is essential for MCM function in vivo. As a control, we tested whether it was possible to introduce a silent mutation into the vicinity of the cysteine 140 codon by changing the sequence GGGACG encoding glycine 141 and threonine 142 to an Acc65I restriction site, GGTACC (mutation $\mathrm{mcm}-\mathrm{S} 1$ ). Sixty colonies were screened by Acc65I digestion of the PCR amplified $\mathrm{mcm}$ gene: 26 contained the Acc65I site (data not shown), indicating that the region of the gene encoding the zinc binding domain can be mutated in this manner.

\section{SENSITIVITY TO MITOMYCIN C}

In total, we isolated 13 mutant strains carrying either single alanine substitutions, paired alanine substitutions and short sequence deletions in two conserved sequence elements, the $\beta 7-\beta 8$ and $\beta 9-\beta 10 \beta$-hairpin loops. None of these strains displayed obvious growth deficiencies: none of the strains grew slowly (Figure 4A) nor were any of the strains cold-sensitive or 


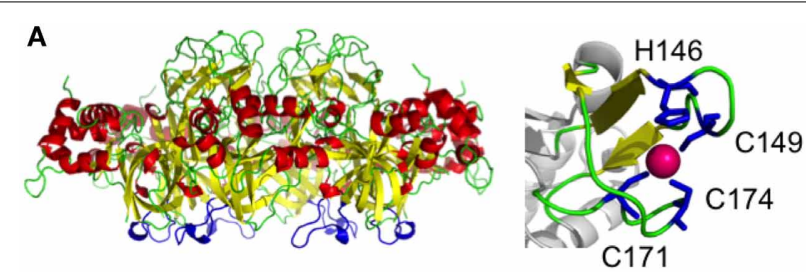

B

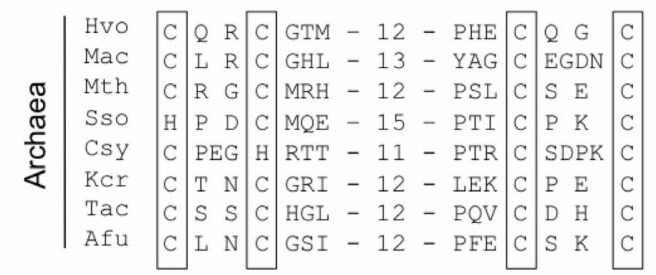

C

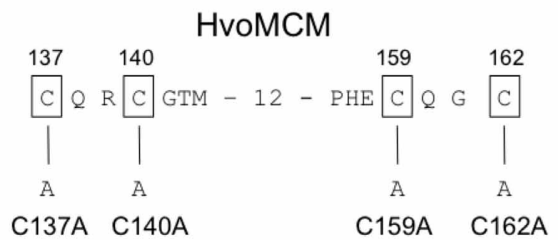

FIGURE 6 | Mutagenesis of the zinc binding domain. (A) Structure of the S. solfataricus MCM N-terminal domain hexamer (PDB 2VL6) with the zinc binding domains highlighted in blue, alongside a close-up view showing the coordination of zinc by the four conserved cysteines. (B) Multiple sequence alignment of the zinc binding domain region from diverse archaeal species (see legend to Figure $\mathbf{3}$ for key and Table $\mathbf{1}$ for strain details and protein accession numbers). Conserved cysteines are shown boxed. (C) Location and nature of intended mutations in Hfx. volcanii MCM protein. Attempts were made to construct four cysteine-to-alanine point mutants. See text for details.

temperature-sensitive (data not shown). We also tested whether the strains might be sensitive to DNA damage induced by the DNA modifying agent mitomycin C (MMC). MMC forms three types of MMC-DNA adducts: monoadducts, intrastrand biadducts and interstrand crosslinks (Tomasz, 1995; Bargonetti et al., 2010). Of the 13 tested strains, one strain, mom-bH5, showed increased sensitivity to MMC treatment (Figure 4B). This mutant has two adjacent residues in the $\beta 9-\beta 10 \beta$-hairpin loop, asparagine 234 and glutamate 235, replaced with alanine.

\section{DISCUSSION}

The MCM helicase is the key catalytic engine of DNA unwinding during chromosome replication in eukaryotes and most likely in archaea also. Three factors make the archaeal MCM proteins excellent models for their human counterparts. First, the relatively high level of sequence similarity between the eukaryotic and archaeal MCM proteins throughout the non-catalytic N-terminal and catalytic AAA+ domains. Second, the relative simplicity of the homohexameric archaeal MCM complexes compared to the heterohexameric eukaryotic complexes. Third, the relative ease with which certain archaeal MCM proteins can be purified in recombinant form, assayed for various activities and crystallized for structure determination. It is striking that although more than 10 years have passed since publication of the first partial crystal structure of an archaeal MCM (Fletcher et al., 2003), no highresolution eukaryotic MCM structures have been solved. Until this occurs, the archaea will remain an important model allowing detailed dissection of MCM protein function.

Using multiple protein sequence alignments and crystal structures as a guide, a number of groups have identified regions of the MCM protein with potentially important roles in MCM function. These have then been mutated and the consequences for MCM activity determined by a variety of in vitro biochemical assays. The $\beta 7-\beta 8 \beta$-hairpin loop was first identified in this way, for example (Sakakibara et al., 2008; Barry et al., 2009). However, despite the growing number of similar studies in the literature, no attempt has been made to examine the in vivo consequences of such mutations.

Here we describe the results of probing the in vivo function of specific amino acids within an archaeal MCM protein using the haloarchaeon $H f x$. volcanii as a model. Hfx. volcanii encodes a single MCM protein of 702 amino acids. This protein is part of the core group of haloarchaeal MCM proteins defined in a previous study (MacNeill, 2009) and expanded upon here. Each of 26 haloarchaeal species investigated encodes a single member of this group (Table 1, Figure 1). Ten species encode additional MCM family proteins classed as outliers. Given that a single core protein is found in all species examined and that 16 of the species encode only a single MCM, it is highly likely that these proteins act as replicative helicases. In support of this, the single MCM protein encoded by Hbt. salinarum NRC-1 (equivalent to the Hbt. salinarum R1 protein listed in Table 1 and labeled as number 4 in Figure 1) has previously been shown to be essential for cell survival (Berquist et al., 2007). While we have not attempted to delete the Hfx. volcanii $\mathrm{mcm}$ gene in its entirety, our inability to isolate certain mutant $\mathrm{mcm}$ alleles in this study strongly points to the $H f x$. volcanii $\mathrm{mcm}$ being essential also.

The cellular functions of the outlying haloarchaeal MCM proteins are unknown. With the exception of the group of four C-terminally truncated MCM proteins highlighted in Figure 1 (proteins labeled 5B, 11C, 17B, and 19B), all the outliers possess intact Walker A, Walker B and arginine finger motifs, suggesting that they may be active as ATPase and/or DNA helicases. Interestingly, T. kodakarensis encodes three MCM proteins, all three of which have helicase activity, although only one is essential for cell viability and which therefore is the likely replicative helicase (Ishino et al., 2011; Pan et al., 2011).

In order to determine whether $H f x$. volcanii presented a workable model for reverse genetic analysis of archaeal MCM function, we targeted three regions of the proteins for investigation. Initially, we focused on the $\beta 7-\beta 8 \beta$-hairpin loop located at the interface between the $\mathrm{N}$-terminal and catalytic domains (Figure 3A) and previously identified as having a role in communicating conformational changes from the DNA binding $\mathrm{N}$ terminal domain to the catalytic AAA+ domain (Sakakibara et al., 2008 ; Barry et al., 2009). The $\beta 7-\beta 8$ loop is well conserved across eukaryotic and archaeal evolution (Figure 3B). In this study, we constructed five strains ( $m c m-r M 1, m c m-r M 3-m c m-r M 6)$ 
carrying single point mutations in the $H f x$. volcanii MCM $\beta 7-\beta 8$ $\beta$-hairpin loop (Table 2, Figures 3, 4). The mutated residues included glutamine 186, which is conserved in both archaeal and eukaryotic MCM proteins (Figure 3B) but which can be replaced by alanine without significantly affecting cell growth, as well as three charged residues (glutamates 190 and 196, and arginine 193) and glutamine 199, all of which were also replaced by alanine without markedly affecting growth rates. In addition, none of the five $\beta 7-\beta 8$ loop mutants led to increased sensitivity to MMC exposure in spotting assays (Figure 4).

In contrast, we were unable to isolate mutant $\mathrm{mcm}$-rM2 encoding a protein in which conserved residue glutamate 187 was replaced by alanine, nor any of the four deletion alleles $\mathrm{mcm}$ $r D 1-m c m-r D 4$. Replacement of glutamate 182 in the M. thermoautotrophicum MCM protein, the residue corresponding to glutamate 187 in Hfx. volcanii, with arginine led to marked reductions in ATPase and helicase activities in vitro, without affecting either ATP or DNA binding (Sakakibara et al., 2008), whereas mutation of conserved glutamine 181 (equivalent to glutamine 186 in Hfx. volcanii MCM, the residue mutated in $m c m-r M 1$ ) to alanine had little impact. Clearly, conservation is no predictor of in vivo essentiality.

We turned next to the $\beta 7-\beta 8 \beta$-hairpin loop, also known as the NT-hairpin. This loop extends into the central channel of the MCM hexamer and may have a role in tracking DNA through the channel based on the fact that mutating two positively charged residues at the tip of the loop in the M. thermoautotrophicum MCM protein abolishes DNA binding in vitro (Fletcher et al., 2003). In $H$. volcanii, only a single basic residue is present in the $\beta 9-\beta 10$ loop region, arginine 236 . This is likely to lie at or near the tip of the $\beta$-hairpin (Figures 5B,C). By comparison with M. thermoautotrophicum, it would be reasonable to predict that this amino acid would be essential for N-terminal domain DNA binding and thus for MCM function in vivo. In the absence of more widespread sequence conservation, we chose to probe the in vivo function of the $\beta 7-\beta 8 \beta$-hairpin loop initially by attempting to construct a series of six mutants in which adjacent amino acids were replaced with pairs of alanines (Figure 5D). All six mutant strains (Table 2, Figures 3, 5) were viable, including $\mathrm{mcm}-b \mathrm{H} 6$ in which arginine 236 was replaced with alanine. Next, we attempted to construct four different $\beta 9-\beta 10$ loop deletion mutants but only two were viable: $m \mathrm{~cm}-\mathrm{hD} 1$ and $m \mathrm{~cm}-\mathrm{hD} 2$ (Table 2, Figures 3, 5). The former removes amino acids 231 and 232, and the latter amino acids 230-233. In contrast, strains carrying two larger deletions, $m c m-h D 3$ and $m c m-h D 4$, removing amino acids 229-234 and 228-235, respectively, could not be isolated. Thus, these results clearly demonstrate that while no individual amino acid within the $\beta 9-\beta 10$ hairpin loop is essential for $H f x$. volcanii MCM function in vivo, the loop does have a key role to play.

In addition to mutagenizing the $\beta 7-\beta 8$ and $\beta 9-\beta 10$ loops, we also attempted to individually replace with alanine each of the four cysteine residues that make up the zinc binding domain (Figure 6). However, we were unable to recover any of four desired mutants, $m \mathrm{~cm}-C 137 \mathrm{~A}, \mathrm{mcm}-\mathrm{C140A}$, $\mathrm{mcm}-\mathrm{C159A}$, or $\mathrm{mcm}-\mathrm{C162A}$, and conclude that the zinc binding domain is therefore likely to have an essential function in vivo. Consistent with this, replacing the equivalent of $H f x$. volcanii MCM cysteine 162 with serine produces an $M$. thermoautotrophicum MCM protein with impaired ATPase and single-stranded DNA binding activities and no helicase activity (Poplawski et al., 2001).

Finally, the mutant $H f x$. volcanii strains generated in this study were tested for increased sensitivity to the DNA modifying drug mitomycin $\mathrm{C}$ (MMC). MMC is a potent DNA interstrand crosslinker and is widely used as replication fork blocking agent, as replication cannot continue past such crosslinks. We tested all 13 viable $\mathrm{mcm}$ alleles for MMC sensitivity by spotting serially diluted cultures onto medium containing increasing concentrations of MMC and found one strain, mcm-bH5, that displayed significantly enhanced sensitivity compared with the parental wild-type H26 (Figure 4B). On medium lacking $\mathrm{MMC}$, growth of $m c m-b H 5$, as with the other viable $\beta 9-\beta 10$ loop mutants, is indistinguishable from wild-type (Figure 4A). The $m c m-b H 5$ protein carries a double substitution in $\beta 9-\beta 10$ loop with asparagine 234 and glutamate 235 being replaced with a pair of significantly less bulky alanines. In the absence of a crystal structure of the $H f x$. volcanii MCM protein, it is difficult to predict whether the impact of the $m c m-b H 5$ mutations is confined to the $\beta 9-\beta 10$ loop alone or whether these amino acid changes will have a wider impact on $\mathrm{N}$-terminal domain structure. It is also difficult to envisage how the $\mathrm{mcm}$ - $b \mathrm{H} 5$ mutations would cause cells to become supersensitive to MMC, particularly as interpreting the effect of MMC on cells is complicated by the different modes of action of this compound. MMC forms at least four different types of DNA adduct: two species of MMC-mono-dG-adduct, intra-strand dG-MMC-dG biadducts and inter-strand dG-MMC-dG crosslinks, with the latter being assumed to be the replication fork blocking lesion (Tomasz, 1995; Bargonetti et al., 2010). Given the key role of the MCM helicase at the fork it is tempting to speculate that the supersensitivity of $\mathrm{mcm}-\mathrm{bH} 5$ is a result of difficulties that arise when the mutant helicase encounters an inter-strand MMC crosslink. However, confirmation of this clearly requires additional experimentation.

In conclusion, the results presented here demonstrate the feasibility of using $H f x$. volcanii as a model system for reverse genetic analysis of archaeal MCM protein function, provide important confirmation of the in vivo importance of conserved structural features identified by previous bioinformatic, biochemical and structural studies, and offer the prospect of more extensive mutational analysis, not only of MCM but of other key replication factors, in the future.

\section{AUTHOR CONTRIBUTIONS}

Tatjana P. Kristensen, Reeja Maria Cherian, and Fiona C. Gray performed the bulk of the experimental work. Stuart A. MacNeill designed the study, carried out some of the experimental work and wrote the manuscript.

\section{ACKNOWLEDGMENTS}

We would like to thank our colleagues in both Copenhagen and St. Andrews for their help with this study. This work was funded by Forskningsrådet for Natur og Univers (FNU sagsnr. 272-050446). 


\section{SUPPLEMENTARY MATERIAL}

The Supplementary Material for this article can be found online at: http://www.frontiersin.org/journal/10.3389/fmicb.2014.00 123/abstract

Supplementary Figure S1 | Sequence analysis of $\beta 7-\beta 8 \beta$-hairpin mutant

strains. Codons mutated to encode alanine are boxed.

\section{Supplementary Figure S2 | Sequence analysis of $\beta 9-\beta 10 \beta$-hairpin mutant}

strains. Codons mutated to encode alanine pairs (mutants bH1-6) are boxed. Vertical lines in hD1 and hD2 indicate location of deleted sequences.

\section{REFERENCES}

Allers, T., Ngo, H. P., Mevarech, M., and Lloyd, R. G. (2004). Development of additional selectable markers for the halophilic archaeon Haloferax volcanii based on the leuB and trpA genes. Appl. Environ. Microbiol. 70, 943-953. doi: 10.1128/AEM.70.2.943-953.2004

Bae, B., Chen, Y. H., Costa, A., Onesti, S., Brunzelle, J. S., Lin, Y., et al. (2009). Insights into the architecture of the replicative helicase from the structure of an archaeal MCM homolog. Structure 17, 211-222. doi: 10.1016/j.str.2008.11.010

Bargonetti, J., Champeil, E., and Tomasz, M. (2010). Differential toxicity of DNA adducts of mitomycin C. J. Nucleic Acids 2010:698960. doi: 10.4061/2010/ 698960

Barry, E. R., Lovett, J. E., Costa, A., Lea, S. M., and Bell, S. D. (2009). Intersubunit allosteric communication mediated by a conserved loop in the MCM helicase. Proc. Natl. Acad. Sci. U.S.A. 106, 1051-1056. doi: 10.1073/pnas.0809192106

Berquist, B. R., Dassarma, P., and Dassarma, S. (2007). Essential and non-essential DNA replication genes in the model halophilic Archaeon, Halobacterium sp. NRC-1. BMC Genet 8:31. doi: 10.1186/1471-2156-8-31

Bitan-Banin, G., Ortenberg, R., and Mevarech, M. (2003). Development of a gene knockout system for the halophilic archaeon Haloferax volcanii by use of the pyrE gene. J. Bacteriol. 185, 772-778. doi: 10.1128/JB.185.3.772-778.2003

Brewster, A. S., Wang, G., Yu, X., Greenleaf, W. B., Carazo, J. M., Tjajadi, M., et al. (2008). Crystal structure of a near-full-length archaeal MCM: functional insights for an AAA+ hexameric helicase. Proc. Natl. Acad. Sci. U.S.A. 105, 20191-20196. doi: 10.1073/pnas.0808037105

Duderstadt, K. E., and Berger, J. M. (2008). AAA+ ATPases in the initiation of DNA replication. Crit. Rev. Biochem. Mol. Biol. 43, 163-187. doi: 10.1080/10409230802058296

Evrin, C., Clarke, P., Zech, J., Lurz, R., Sun, J., Uhle, S., et al. (2009). A doublehexameric MCM2-7 complex is loaded onto origin DNA during licensing of eukaryotic DNA replication. Proc. Natl. Acad. Sci. U.S.A. 106, 20240-20245. doi: 10.1073/pnas.0911500106

Farkas, J. A., Picking, J. W., and Santangelo, T. J. (2013). Genetic techniques for the archaea. Annu. Rev. Genet. 47, 539-561. doi: 10.1146/annurev-genet-111212133225

Fletcher, R. J., Bishop, B. E., Leon, R. P., Sclafani, R. A., Ogata, C. M., and Chen, X. S. (2003). The structure and function of MCM from archaeal $M$. thermoautotrophicum. Nat. Struct. Biol. 10, 160-167. doi: 10.1038/nsb893

Fu, Y., Slaymaker, I. M., Wang, J., Wang, G., and Chen, X. S. (2014). The 1.8-a crystal structure of the N-terminal domain of an archaeal MCM as a right-handed filament. J. Mol. Biol. 426, 1512-1523. doi: 10.1016/j.jmb.2013.12.025

Gambus, A., Khoudoli, G. A., Jones, R. C., and Blow, J. J. (2011). MCM2-7 form double hexamers at licensed origins in Xenopus egg extract. J. Biol. Chem. 286, 11855-11864. doi: 10.1074/jbc.M110.199521

Gogarten, J. P., and Hilario, E. (2006). Inteins, introns, and homing endonucleases: recent revelations about the life cycle of parasitic genetic elements. BMC Evol. Biol. 6:94. doi: 10.1186/1471-2148-6-94

Hawkins, M., Malla, S., Blythe, M. J., Nieduszynski, C. A., and Allers, T. (2013). Accelerated growth in the absence of DNA replication origins. Nature 503, 544-547. doi: 10.1038/nature 12650

Ishino, S., Fujino, S., Tomita, H., Ogino, H., Takao, K., Daiyasu, H., et al. (2011). Biochemical and genetical analyses of the three $\mathrm{mcm}$ genes from the hyperthermophilic archaeon, Thermococcus kodakarensis. Genes Cells 16, 1176-1189. doi: 10.1111/j.1365-2443.2011.01562.x

Kamada, K. (2012). The GINS complex: structure and function. Subcell. Biochem. 62, 135-156. doi: 10.1007/978-94-007-4572-8_8
Krastanova, I., Sannino, V., Amenitsch, H., Gileadi, O., Pisani, F. M., and Onesti, S. (2012). Structural and functional insights into the DNA replication factor Cdc45 reveal an evolutionary relationship to the DHH family of phosphoesterases. J. Biol. Chem. 287, 4121-4128. doi: 10.1074/jbc.M111.285395

Larkin, M. A., Blackshields, G., Brown, N. P., Chenna, R., Mcgettigan, P. A., McWilliam, H., et al. (2007). Clustal W and Clustal X version 2.0. Bioinformatics 23, 2947-2948. doi: 10.1093/bioinformatics/btm404

Li, Z., Pan, M., Santangelo, T. J., Chemnitz, W., Yuan, W., Edwards, J. L., et al. (2011). A novel DNA nuclease is stimulated by association with the GINS complex. Nucleic Acids Res. 39, 6114-6123. doi: 10.1093/nar/gkr181

Liu, W., Pucci, B., Rossi, M., Pisani, F. M., and Ladenstein, R. (2008). Structural analysis of the Sulfolobus solfataricus MCM protein N-terminal domain. Nucleic Acids Res. 36, 3235-3243. doi: 10.1093/nar/gkn183

MacNeill, S. A. (2009). The haloarchaeal chromosome replication machinery. Biochem. Soc. Trans. 37, 108-113. doi: 10.1042/BST0370108

MacNeill, S. A. (2010). Structure and function of the GINS complex, a key component of the eukaryotic replisome. Biochem. J. 425, 489-500. doi: 10.1042/BJ20091531

Magrane, M., and UniProt Consortium. (2011). UniProt Knowledgebase: a hub of integrated protein data. Database (Oxford) 2011:bar009. doi: 10.1093/database/ bar009

Makarova, K. S., Koonin, E. V., and Kelman, Z. (2012). The CMG (CDC45/RecJ, MCM, GINS) complex is a conserved component of the DNA replication system in all archaea and eukaryotes. Biol. Direct 7:7. doi: 10.1186/1745-6150-7-7

Marinsek, N., Barry, E. R., Makarova, K. S., Dionne, I., Koonin, E. V., and Bell, S. D. (2006). GINS, a central nexus in the archaeal DNA replication fork. EMBO Rep. 7, 539-545. doi: 10.1038/sj.embor.7400649

Morgunova, E., Gray, F. C., Macneill, S. A., and Ladenstein, R. (2009). Structural insights into the adaptation of proliferating cell nuclear antigen (PCNA) from Haloferax volcanii to a high-salt environment. Acta Crystallogr. D Biol. Crystallogr. 65, 1081-1088. doi: 10.1107/S0907444909 029321

Norais, C., Hawkins, M., Hartman, A. L., Eisen, J. A., Myllykallio, H., and Allers, T. (2007). Genetic and physical mapping of DNA replication origins in Haloferax volcanii. PLoS Genet. 3:e77. doi: 10.1371/journal.pgen.0030077

Onesti, S., and MacNeill, S. A. (2013). Structure and evolutionary origins of the CMG complex. Chromosoma 122, 47-53. doi: 10.1007/s00412-013-0397-x

Oyama, T., Ishino, S., Fujino, S., Ogino, H., Shirai, T., Mayanagi, K., et al. (2011). Architectures of archaeal GINS complexes, essential DNA replication initiation factors. BMC Biol. 9:28. doi: 10.1186/1741-7007-9-28

Pan, M., Santangelo, T. J., Li, Z., Reeve, J. N., and Kelman, Z. (2011). Thermococcus kodakarensis encodes three MCM homologs but only one is essential. Nucleic Acids Res. 39, 9671-9680. doi: 10.1093/nar/gkr624

Perler, F. B. (2002). InBase: the intein database. Nucleic Acids Res. 30, 383-384. doi: $10.1093 /$ nar/30.1.383

Perriere, G., and Gouy, M. (1996). WWW-query: an on-line retrieval system for biological sequence banks. Biochimie 78, 364-369. doi: 10.1016/03009084(96)84768-7

Poidevin, L., and MacNeill, S. A. (2006). Biochemical characterisation of LigN, an $\mathrm{NAD}^{+}$-dependent DNA ligase from the halophilic euryarchaeon Haloferax volcanii that displays maximal in vitro activity at high salt concentrations. BMC Mol. Biol. 7:44. doi: 10.1186/1471-2199-7-44

Poplawski, A., Grabowski, B., Long, S. E., and Kelman, Z. (2001). The zinc finger domain of the archaeal minichromosome maintenance protein is required for helicase activity. J. Biol. Chem. 276, 49371-49377. doi: 10.1074/jbc.M108 519200

Remus, D., Beuron, F., Tolun, G., Griffith, J. D., Morris, E. P., and Diffley, J. F. (2009). Concerted loading of $\mathrm{Mcm} 2-7$ double hexamers around DNA during DNA replication origin licensing. Cell 139, 719-730. doi: 10.1016/j.cell.2009.10.015

Sakakibara, N., Kasiviswanathan, R., Melamud, E., Han, M., Schwarz, F. P., and Kelman, Z. (2008). Coupling of DNA binding and helicase activity is mediated by a conserved loop in the MCM protein. Nucleic Acids Res. 36, 1309-1320. doi: $10.1093 / \mathrm{nar} / \mathrm{gkm} 1160$

Sanchez-Pulido, L., and Ponting, C. P. (2011). Cdc45: the missing RecJ ortholog in eukaryotes? Bioinformatics 27, 1885-1888. doi: 10.1093/bioinformatics/btr332

Skowyra, A., and MacNeill, S. A. (2012). Identification of essential and nonessential single-stranded DNA-binding proteins in a model archaeal organism. Nucleic Acids Res. 40, 1077-1090. doi: 10.1093/nar/gkr838 
Slaymaker, I. M., and Chen, X. S. (2012). MCM structure and mechanics: what we have learned from archaeal MCM. Subcell. Biochem. 62, 89-111. doi: 10.1007/978-94-007-4572-8_6

Slaymaker, I. M., Fu, Y., Toso, D. B., Ranatunga, N., Brewster, A., Forsburg, S. L., et al. (2013). Mini-chromosome maintenance complexes form a filament to remodel DNA structure and topology. Nucleic Acids Res. 41, 3446-3456. doi: 10.1093/nar/gkt022

Stroud, A., Liddell, S., and Allers, T. (2012). Genetic and biochemical identification of a novel single-stranded DNA-binding complex in Haloferax volcanii. Front. Microbiol. 3:224. doi: 10.3389/fmicb.2012.00224

Tomasz, M. (1995). Mitomycin C: small, fast and deadly (but very selective). Chem. Biol. 2, 575-579. doi: 10.1016/1074-5521(95)90120-5

Vijayraghavan, S., and Schwacha, A. (2012). The eukaryotic Mcm2-7 replicative helicase. Subcell. Biochem. 62, 113-134. doi: 10.1007/978-94-007-4572-8_7

Walters, A. D., and Chong, J. P. (2010). An archaeal order with multiple minichromosome maintenance genes. Microbiology 156, 1405-1414. doi: 10.1099/mic.0.036707-0

Wendoloski, D., Ferrer, C., and Dyall-Smith, M. L. (2001). A new simvastatin (mevinolin)-resistance marker from Haloarcula hispanica and a new Haloferax volcanii strain cured of plasmid pHV2. Microbiology 147, 959-964.

Wiedemann, C., Ohlenschläger, O., Medagli, B., Onesti, S., and Görlach, M. (2013) ${ }^{1} \mathrm{H},{ }^{15} \mathrm{~N}$ and ${ }^{13} \mathrm{C}$ chemical shift assignments for the winged helix domains of two archeal MCM C-termini. Biomol. NMR Assign. doi: 10.1007/s12104-0139516-0. [Epub ahead of print].

Winter, J. A., Christofi, P., Morroll, S., and Bunting, K. A. (2009). The crystal structure of Haloferax volcanii proliferating cell nuclear antigen reveals unique surface charge characteristics due to halophilic adaptation. BMC Struct. Biol. 9:55. doi: 10.1186/1472-6807-9-55
Yuan, H., Liu, X.-P., Han, Z., Allers, T., Hou, J.-L., and Liu, J.-H. (2013). RecJ-like protein from Pyrococcus furiosus has $3^{\prime}-5^{\prime}$ exonuclease activity on RNA: implications for proofreading of $3^{\prime}$-mismatched RNA primers in DNA replication. Nucleic Acid Res. 41, 5817-5826. doi: 10.1093/nar/ gkt275

Zhao, A., Gray, F. C., and MacNeill, S. A. (2006). ATP- and NAD ${ }^{+}$-dependent DNA ligases share an essential function in the halophilic archaeon Haloferax volcanii. Mol. Microbiol. 59, 743-752. doi: 10.1111/j.1365-2958.2005.04975.x

Conflict of Interest Statement: The authors declare that the research was conducted in the absence of any commercial or financial relationships that could be construed as a potential conflict of interest.

Received: 09 February 2014; accepted: 10 March 2014; published online: 26 March 2014.

Citation: Kristensen TP, Maria Cherian R, Gray FC and MacNeill SA (2014) The haloarchaeal MCM proteins: bioinformatic analysis and targeted mutagenesis of the $\beta 7-\beta 8$ and $\beta$ 9- $\beta 10$ hairpin loops and conserved zinc binding domain cysteines. Front. Microbiol. 5:123. doi: 10.3389/fmicb.2014.00123

This article was submitted to Extreme Microbiology, a section of the journal Frontiers in Microbiology.

Copyright (c) 2014 Kristensen, Maria Cherian, Gray and MacNeill. This is an open-access article distributed under the terms of the Creative Commons Attribution License (CC BY). The use, distribution or reproduction in other forums is permitted, provided the original author(s) or licensor are credited and that the original publication in this journal is cited, in accordance with accepted academic practice. No use, distribution or reproduction is permitted which does not comply with these terms. 